\title{
Los términos de género natural: ¿Ha malinterpretado Kripke la teoría de Mill?*
}

\section{(Natural Kind Terms: Has Kripke Misinterpreted Mill's Theory?)}

\author{
Luis FERnÁNDEZ MORENO \\ Universidad Complutense de Madrid \\ luis.fernandez@filos.ucm.es
}

Recibido: 9 de septiembre de 2010

Aceptado: 13 de octubre de 2010

\section{Resumen}

En un famoso pasaje de Naming and Necessity Kripke sintetiza el núcleo de su crítica a la teoría descriptiva de los términos de género natural, tomando en consideración la teoría de los términos generales propuesta por Mill, en tanto que aplicada a los términos de género natural, como paradigma de ese tipo de teoría. El objetivo de este artículo es argüir que la teoría genérica de Mill acerca de los términos generales no coincide con su teoría sobre ese tipo de términos generales que son los términos de género natural y que la principal tesis de esta última no está sujeta a las objeciones que Kripke presenta en el pasaje mencionado.

Palabras clave: teoría descriptiva, connotación, términos generales, géneros naturales, términos de género natural.

\begin{abstract}
In a famous passage of Naming and Necessity Kripke summarizes the core of his criticism to the description theory of natural kind terms, taking into account the the-

\footnotetext{
* Agradezco las observaciones de un evaluador anónimo de la Revista de Filosofía a una versión preliminar de este escrito. La elaboración de este artículo ha sido financiada por el Ministerio de Ciencia e Innovación, en el marco del proyecto FFI2008-03092, así como por la ayuda BSCH-UCM concedida al grupo de investigación 930174 ("Filosofía del Lenguaje, de la Naturaleza y de la Ciencia") en la convocatoria 2010 de grupos consolidados.
} 
ory of general terms proposed by Mill, insofar as it is applied to natural kind terms, as a paradigm of that sort of theory. The aim of this paper is to argue that Mill's generic theory on general terms does not coincide with his theory concerning the sort of general terms that natural kind terms are and that the main thesis of the latter is not subject to Kripke's objections put forward in the aforementioned passage.

Keywords: description theory, connotation, general terms, natural kinds, natural kind terms.

\section{Términos de género natural: la interpretación de la teoría de Mill por parte de Kripke}

Uno de los tipos de expresiones que han estado en el centro del debate entre la teoría descriptiva y la teoría causal son los términos de género natural. Las consideraciones de S. Kripke sobre los términos de género natural en (1980) son secundarias con respecto a las correspondientes a los nombres propios. Sólo tras haberse ocupado de los nombres propios - en las dos primeras conferencias de (1980) Kripke atiende, en la tercera y última conferencia de (1980), a los términos de género natural, y alega la existencia de similitudes entre los términos de género natural y los nombres propios. A la base de esas semejanzas se encuentra la tesis de que la teoría descriptiva no es adecuada ni con respecto a los nombres propios ni con respecto a los términos de género natural. Uno de los objetivos principales de Kripke en (1980) consiste en criticar la teoría descriptiva y esbozar una teoría de la referencia alternativa, que ha pasado a ser denominada "causal" o "histórico-causal", aunque no es mi objetivo en este artículo entrar en los detalles de la caracterización positiva de este tipo de teoría - véase, por ejemplo, Fernández Moreno (2006), cap. IV -. La crítica a la teoría descriptiva de los nombres propios es llevada a cabo en las dos primeras conferencias de (1980), mientras que las objeciones contra la teoría descriptiva de los términos de género natural son formuladas en la tercera conferencia.

En su crítica a la teoría descriptiva de los nombres propios Kripke considera que esta teoría puede concebirse como una teoría del significado $o$ como una teoría de la referencia (véase, por ejemplo, Kripke 1980, pp. 32-3, 53-4 y 59). Esta distinción concuerda con el proceder de Kripke en su crítica a la teoría descriptiva de los nombres propios, pues él presenta sus objeciones contra la teoría descriptiva de este tipo de expresiones, concebida como una teoría del significado, preferentemente en la conferencia primera de (1980), mientras que formula sus argumentos contra la teoría descriptiva de los nombres propios, concebida como una teoría de la referencia, principalmente en la conferencia segunda de (1980). En la tercera conferencia se presentan objeciones contra la teoría descriptiva en sus dos versiones en tanto que aplicada a los términos de género natural. 
Conviene señalar que Kripke, como la mayoría de los autores, no presenta una caracterización precisa de los términos de género natural, limitándose básicamente a afirmar que los términos de género natural son términos generales y que entre ellos se encuentran términos contables, como "gato" o "tigre", y términos de masa, como "agua" y "oro" - Kripke (1980), p. 134; véase también p. 127 -. Si a esto unimos que Kripke emplea indistintamente las expresiones "términos de género natural" (natural kind terms) y "términos de géneros naturales" (terms for natural kinds) - véase Kripke 1980, p. 127 -, habría que concluir que los términos de género natural son caracterizados por Kripke simplemente como términos generales que designan o, al menos, pretenden designar géneros naturales, como los géneros gato o agua - acerca de la concepción de Kripke sobre los géneros naturales véase la sección 3. infra-.

El núcleo de la crítica de Kripke a la teoría descriptiva de ese tipo de términos generales que son los términos de género natural se encuentra en un famoso pasaje de la tercera conferencia de (1980), del que extraemos los aspectos más relevantes desde el punto de vista de su oposición a la teoría de J.S. Mill:

Es interesante comparar mis tesis con las de Mill. Mill dice que todos los nombres 'generales' son connotativos; un predicado tal como 'ser humano' se define como la conjunción de ciertas propiedades que proporcionan condiciones necesarias y suficientes para la humanidad - la racionalidad, la animalidad y ciertos rasgos físicos - . La tradición lógica moderna, como representada por Frege y Russell, parece sostener que Mill $[\ldots]$ acertaba con respecto a los nombres generales. La filosofía más reciente ha permanecido fiel a esto, excepto que [...] reemplaza con frecuencia la noción de propiedades definitorias por la de cúmulo de propiedades, de las cuales sólo algunas tienen que satisfacerse en cada caso particular. Mi propia posición [...] considera que Mill [...] se equivocaba con respecto a los nombres 'generales' [...] En un sentido importante, los términos generales tales como 'vaca' y 'tigre' no expresan propiedades, a menos que ser una vaca cuente trivialmente como una propiedad. Ciertamente [los términos] 'vaca' y 'tigre' no son, como Mill pensó, abreviaturas de la conjunción de propiedades mediante las cuales los podría definir un diccionario (Kripke 1980, pp. 127-128; véase en un sentido similar 1980, pp. 134-135). ${ }^{1}$

\footnotetext{
${ }^{1}$ Con respecto a la teoría de Frege acerca de los términos generales y, más precisamente, de los términos que Frege denominó "conceptuales", véase mi (2009). Por lo que concierne a la teoría de Russell conviene indicar que él afirma en (1912) que "[1]as palabras comunes [...] son generalmente en realidad descripciones" (Russell 1912, p. 29) y que una descripción (definida) expresa una propiedad (ibíd.) o una serie de propiedades. Por otra parte, la "filosofía más reciente" con respecto a los términos generales a la que Kripke alude en dicho pasaje resultaría de la extensión a este tipo de términos de una teoría como la propuesta por Searle en (1969) acerca de los nombres propios.
} 
En este pasaje Kripke caracteriza la teoría de Mill de modo que este autor habría sostenido acerca de los términos generales, y en especial acerca de los términos de género natural - como, por ejemplo, "vaca", "tigre" o "ser humano" -, que un término de este tipo viene definido mediante la conjunción de una serie de propiedades, que constituyen la connotación del término, y dicha conjunción de propiedades proporcionaría condiciones necesarias y suficientes para la referencia del término. Ahora bien, Kripke considera que dicha teoría, y en general la teoría descriptiva de los términos generales, de la que la teoría de Mill sería paradigmática, no es adecuada para ese tipo de términos generales que son los términos de género natural.

Haciéndonos eco de la afirmación de Kripke de que "[e]s interesante comparar mis tesis con las de Mill", tras exponer la teoría genérica de Mill acerca de los términos generales, alegaré que esta teoría no coincide con la que él propone acerca de ese tipo de términos generales que son los términos de género natural y que la tesis principal de esta última no está sujeta a las objeciones que Kripke presenta en el pasaje citado.

\section{La teoría de Mill acerca de los términos generales}

Aunque Kripke no indica explícitamente la página de Mill (1843) donde se encuentra el primer ejemplo de término general al que alude en el texto anterior, el pasaje en cuestión parece ser el siguiente, con la ligera diferencia de que en él se emplea el término "hombre" y no el de "ser humano":

La palabra hombre [...] denota a Pedro, a Juana, a Juan y a un número indeterminado de otros individuos, de los que, considerados como una clase, es el nombre. Pero se aplica a ellos, porque poseen, y para significar que poseen, ciertos atributos. Éstos parecen ser corporeidad, vida animal, racionalidad y una cierta forma externa, que para distinguirla llamamos humana. Toda cosa existente que poseyese todos estos atributos sería llamado un hombre, y cualquier cosa que no poseyese ninguno de ellos, o sólo uno, o dos, o incluso tres de ellos, sin el cuarto, no sería llamado así (Mill 1843, p. 31).

La tesis de Mill acerca de los nombres o términos generales que cabe extraer de este pasaje es que un término general significa un atributo o una serie de atributos de tal manera que los individuos denotados o referidos por el término general serán aquellos que posean todos los atributos significados por el mismo. A este respecto conviene añadir que Mill clasifica los atributos en las categorías de cualidad, relación y cantidad (Mill 1843, pp. 65 ss.) y que en ocasiones Mill emplea indistintamente las expresiones "atributo" y "propiedad" (véase, por ejemplo, Mill 1843, p. 121), siendo este último término del que Kripke se sirve en su caracterización de la teoría descriptiva, incluyendo de manera paradigmática la teoría de Mill, en el texto 
citado anteriormente. No obstante, una adecuada comprensión de la tesis de Mill sobre los nombres o términos generales que acabamos de mencionar requiere que hagamos algunas consideraciones acerca de la teoría de Mill concerniente a este tipo de nombres o términos.

Para expresar la relación existente entre un nombre y su referente Mill suele emplear la expresión "denotación". Él expone su teoría de los nombres generales y de su denotación al hilo de su presentación de las divisiones de los nombres. En el capítulo II, "Acerca de los nombres", del libro I de (1843), titulado "Acerca de los nombres y las proposiciones", Mill presenta una serie de divisiones de los nombres. Las divisiones más relevantes para nuestro objetivo son las existentes entre nombres generales y singulares (o individuales) y entre nombres connotativos y no-connotativos.

Mill formula la división de los nombres en generales y singulares (o individuales) de la siguiente manera:

Un nombre general es [...] aquel que es capaz de ser afirmado con verdad, en el mismo sentido, de cada una de un número indefinido de cosas. Un nombre individual o singular es aquel que sólo es capaz de ser afirmado con verdad, en el mismo sentido, de una cosa (Mill 1843, p.28).

Esta distinción entre nombres generales y singulares (o individuales) podría parafrasearse de manera aproximada en una terminología más actual indicando que los términos generales son capaces de denotar - o de aplicarse a - cada uno de los objetos o individuos de una clase o un conjunto 2 de individuos, mientras que los términos singulares sólo son capaces de denotar un objeto o individuo. Sin embargo, hay, al menos, dos elementos dignos de mención presentes en la definición de Mill de los nombres generales y de los nombres singulares, recién citada, que quedan fuera de esta paráfrasis. En primer lugar, de acuerdo con dicha definición, los individuos denotados por un nombre son aquellos de los que el nombre puede afirmarse con verdad, es decir, de los que puede predicarse con verdad. Esto se debe a que Mill concibe la denotación de los nombres como determinada por la predicación; así él afirma que "un nombre sólo puede decirse que designa o que es un nombre de las cosas de las que puede ser predicado" (Mill 1843, p. 30). En segundo lugar, en la definición de Mill se puntualiza que la afirmación o la predicación (con verdad) ha de ser en el mismo sentido. Esta apelación es claramente relevante en el caso de los nombres o términos generales, lo que puede mostrarse con uno de los ejemplos de nombres generales mencionados por Mill, el término "hombre", al que ya aludimos anteriormente. Mill afirma acerca de este término en otro pasaje lo siguiente:

\footnotetext{
2 En lo siguiente emplearé los términos "clase" y "conjunto" como intercambiables; véase la nota 9 infra.
} 
[...] hombre es capaz de ser verdaderamente afirmado de Juan, Jorge, María y de otras personas [...], y es afirmado de todas ellas en el mismo sentido, pues la palabra hombre expresa ciertas cualidades, y cuando la predicamos de esas personas, afirmamos que todas ellas poseen esas cualidades (Mill 1843, p. 28).

Un nombre es afirmado en un sentido determinado en la medida en que el nombre es usado para expresar ciertas cualidades o, en general, atributos que determinan cuál es la denotación del nombre; los individuos denotados por el nombre serán aquellos que posean los todos los atributos en cuestión, y es de estos individuos de los que el nombre puede ser afirmado con verdad en un sentido determinado. De manera general, un término es afirmado de un individuo en un sentido determinado cuando el término expresa algunos atributos - al menos, un atributo - y al predicarlo del individuo afirmamos que posee esos atributos.

Otra de las divisiones de los nombres propuesta por Mill y relevante para nuestras consideraciones es la existente entre nombres connotativos y no-connotativos. 3 Mill define estos dos tipos de nombres o términos de la siguiente manera:

Un término no-connotativo es aquel que significa sólo un sujeto o sólo un atributo. Un término connotativo es aquel que denota un sujeto e implica un atributo. Por un sujeto se entiende aquí cualquier cosa que posea atributos. (Mill 1843, p. 31).

Ahora bien, como ocurre frecuentemente con las definiciones de los tipos de nombres que Mill presenta, los ejemplos que siguen a dichas definiciones permiten perfilar mejor la definición en cuestión. En este caso los ejemplos puestos por Mill para ilustrar la distinción entre términos connotativos y no-connotativos llevarían a la siguiente caracterización. Un término no-connotativo es aquel que denota sólo un individuo o sólo un atributo, ${ }^{4}$ mientras que un término connotativo es aquel que denota un individuo o una serie de individuos (cada uno de los individuos de un conjunto de individuos) $e$ implica - o involucra o indica, términos empleados indistintamente por Mill - un atributo o una serie de atributos (cada uno de los atributos

\footnotetext{
3 Una de las divisiones de los nombres formulada por Mill a la que no hemos aludido es la división entre nombres concretos y abstractos, que puede formularse de la siguiente manera: un nombre concreto es aquel que denota un objeto o individuo, o una serie de objetos o individuos - cada uno de los objetos o individuos de un conjunto de objetos o individuos -, mientras que un nombre abstracto es aquel que denota un atributo, o una serie de atributos - cada uno de los atributos de un conjunto de atributos -. Una presentación más exhaustiva de la teoría de los nombres de Mill puede consultarse en mi (2006).

4 En el texto de Mill recién citado se caracterizan los términos no-connotativos como aquellos que significan sólo un sujeto o sólo un atributo. Puesto que Mill identifica el significado, en el sentido estricto de esta noción, con la connotación - véase la nota 6 infra-, en dicho texto habría que entender por "significar" denotar; obviamente, los términos no-connotativos, como su misma denominación indica, carecen de connotación.
} 
de un conjunto de atributos). La connotación es así una relación entre un término y un atributo o una serie de atributos. Con respecto a los nombres o términos connotativos, la relación existente entre denotación y connotación radica en que su connotación determina su denotación, pues el individuo o los individuos denotados por un nombre connotativo son aquellos que poseen el atributo o los atributos que constituyen su connotación, mientras que su denotación no determina su connotación, pues puede haber nombres connotativos que poseen la misma denotación, pero distinta connotación.

A tenor de nuestras observaciones sobre la división de los nombres o términos en generales y singulares, que se solapa con la división en connotativos y no-connotativos, los nombres generales y las descripciones definidas son términos connotativos, mientras que, según Mill, los nombres propios son términos no-connotativos. ${ }^{5}$

De acuerdo con lo anterior, cabe atribuir a Mill la tesis de que un nombre o término general es definido mediante un atributo o mediante - la conjunción de - una serie de atributos, a saber, los que constituyen la connotación del término - es decir, los que proporcionan el significado ${ }^{6}$ del término -, de tal manera que los individuos denotados por el término general serán aquellos individuos que posean todos los atributos que figuran en la definición del término general; la conjunción de dichos atributos o propiedades proporciona condiciones necesarias y suficientes para la referencia o denotación del término. Ésta es justamente la tesis acerca de los términos generales que Kripke atribuye a Mill en el pasaje citado anteriormente. No obstante, y como veremos a continuación, esta tesis no coincide con la teoría de Mill acerca de ese tipo de términos generales que son los términos de género natural, y esto a pesar de que para ilustrar dicha tesis en relación con los términos generales Mill se haya servido, como se observó anteriormente, de un término de género natural, como el término "hombre".

\section{Kripke y Mill: géneros naturales y términos de género natural}

Tanto Mill como Kripke han formulado una teoría de los géneros naturales, aunque Mill suele denominarlos "Géneros reales". Es digno de mención que Kripke no ha tomado en consideración, al menos explícitamente, la teoría de Mill acerca de los Géneros reales, aunque para unificar esa terminología con la que hemos empleado

\footnotetext{
5 A pesar de que, como vimos, Kripke discrepa de la teoría de Mill acerca de los términos generales $\mathrm{y}$, en especial, en tanto que aplicada a ese tipo de términos generales que son los términos de género natural, Kripke muestra cierto acuerdo con la teoría de Mill acerca de los términos singulares, como los nombres propios y las descripciones definidas - véase Kripke (1980), pp. 127 y 135 -.

${ }^{6}$ Mill identifica el significado de un término, en el sentido estricto de la palabra "significado", con la connotación del término, de lo que se seguiría que términos carentes de connotación, como según Mill son los nombres propios, habrían de carecer de significado, y esto es algo que Mill literalmente afirma - véase Mill (1843), pp. 34, 133 y 136 -.
} 
hasta aquí seguiré usando la expresión "géneros naturales", a la que Mill también recurre en ocasiones para aludir a los Géneros reales (véase Mill 1843, p. 126).

Aunque hay varias diferencias entre las teorías de los géneros naturales $y$, por tanto, de la referencia de los términos de género natural formuladas por Kripke y por Mill, en lo siguiente sólo atenderé a dos de estas diferencias.

La primera diferencia, y menos importante, radica en el diferente estatuto ontológico que estos autores atribuyen a los géneros naturales. Mill concibe los géneros $\mathrm{y}$, por tanto, los géneros naturales como un tipo de clases (Mill 1843, pp. 122 s.), mientras que la posición de Kripke al respecto es menos clara. En lo concerniente a esta cuestión conviene tomar en consideración el siguiente pasaje de Kripke:

Creo que, en general, la referencia de los términos de géneros naturales (por ejemplo, de géneros animales, vegetales y químicos) se fija de esta manera: la sustancia es definida como el género instanciado por (casi la totalidad) de una muestra dada (Kripke 1980, pp. 135-136; mi cursiva). ${ }^{7}$

En un sentido similar, Kripke alude al caso concreto de la fijación de la referencia del término de género natural "oro", que podríamos imaginar que se habría llevado a cabo mediante un proceder similar al siguiente:

Oro [es decir, la referencia del término 'oro'] es la sustancia instanciada por las cosas que están allí o al menos por casi todas ellas (Kripke 1980, p. 135; mi cursiva). ${ }^{8}$

En estos pasajes Kripke identifica la referencia de un término de género natural $\mathrm{y}$, por tanto, el género natural en cuestión con un tipo de entidad abstracta o universal - aunque no entra en mayores detalles al respecto -, lo que concuerda con su caracterización en los dos pasajes recién citados de la relación entre un género natural y las entidades del mismo como una relación de instanciación o ejemplificación Sin embargo, esta diferencia en el estatuto ontológico de los géneros naturales entre las teorías de Mill y de Kripke queda un tanto desdibujada por el hecho de que este último afirma en algunos pasajes que la relación entre las entidades de un género

\footnotetext{
${ }^{7}$ Con respecto a este texto conviene hacer dos observaciones. Por una parte, Kripke distingue "entre las 'definiciones' que fijan una referencia y las que dan un sinónimo" (Kripke 1980, p. 57); en el texto citado se está entendiendo la expresión "definición" sólo en el primer sentido. Por otra parte, en dicho pasaje Kripke emplea el término "sustancia" como intercambiable con el de "género natural".

${ }^{8}$ La puntualización contenida en este pasaje y en el precedente de Kripke - "casi la totalidad" y "casi todas" - se debe a que algunas de las entidades pertenecientes a la muestra involucrada en la introducción de un término de género natural pueden no ser instancias del género. Si se constata que el número de tales entidades no es reducido, siendo la muestra, por tanto, poco uniforme, puede haber distintas reacciones al respecto, entre las que Kripke menciona las de considerar que la muestra instancia dos tipos de género o la de retirar el término de género natural que suponíamos haber introducido (Kripke 1980, p. 136); en lo siguiente pasaré por alto dicha puntualización.
} 
natural y el género natural en cuestión es la de pertenencia (véase Kripke 1980, pp. 121, 136 y 137), lo que llevaría a suponer que por un género natural y, por tanto, por la referencia de un término de género natural se está entendiendo su extensión, es decir, la clase o el conjunto de las entidades a las que el término se aplica y que, por tanto, pertenecen a ese conjunto. Ahora bien, si se concibe la referencia de un término de género natural como un conjunto de individuos, es difícilmente sostenible la tesis de Kripke de que los términos de género natural son designadores rígidos, es decir, términos que designan la misma entidad con respecto a todos los mundos posibles o, al menos, con respecto a todos los mundos posibles en los que exista la entidad en cuestión, pues los miembros de un género natural podrán ser diferentes en distintos mundos posibles, por lo cual también será diferente el conjunto de tales individuos y, consecuentemente, el género natural en cuestión. Por este motivo, la mayoría de los autores que sostienen esa tesis abogan por la concepción del referente de un término de género natural como un tipo de entidad abstracta o universal instanciada en entidades concretas, que es la concepción más acorde con los dos últimos pasajes citados de Kripke - un autor que mantiene una concepción de este tipo es N. Salmon; véase, por ejemplo, Salmon (2005) -.

No obstante, puesto que en el contexto de la discusión entre Kripke y Mill nada fundamental depende de la elección entre esas dos opciones acerca del estatuto ontológico de los géneros naturales, en lo siguiente supondremos que un género natural y, por tanto, el referente de un término de género natural, es concebido como una clase o un conjunto de individuos, ${ }^{9}$ lo que nos permitirá comparar la concepción más específica de estos autores acerca de los géneros naturales, en la que radica la segunda, y más importante, diferencia, entre sus posiciones.

La concepción de Kripke acerca de los géneros naturales puede formularse en base a las tres tesis siguientes. En primer lugar, entre los miembros de un género natural se encuentra un conjunto de entidades a las que se atribuye el carácter de miembros paradigmáticos del género. En segundo lugar, la atribución de este rasgo a dichas entidades se debe a que comparten una serie de propiedades macroscópicas concernientes principalmente a su apariencia externa, a las que aludiremos como propiedades superficiales. En tercer lugar, suponemos que dichas entidades comparten una estructura interna o, en general, propiedades estructurales (inter-

\footnotetext{
9 Como hemos indicado, los términos de género natural son considerados por Kripke y por Mill como términos generales. Ahora bien, en el caso de Mill se plantea un problema terminológico, pues en relación a la referencia de los términos generales, o en la terminología preferida por Mill de su denotación, él oscila entre dos concepciones distintas. La primera es considerar que lo denotado por un término general son individuos, a saber, los miembros de una clase de individuos (Mill 1843, p. 28; véase también p. 93) - en esta acepción la noción de denotación es equivalente a la noción actual de aplicación de un término -, mientras que en otras ocasiones Mill afirma que lo denotado es la clase en cuestión (véase, por ejemplo, Mill 1843, pp. 118 s.). Aunque en la sección 2 hemos elegido la primera alternativa, en esta sección 3 optaremos preferentemente por la segunda, pues, como acabamos de indicar, vamos a considerar los géneros naturales como clases o conjuntos de individuos.
} 
nas), que subyacen a las propiedades superficiales y de las que éstas dependen; en el caso de las porciones de agua la propiedad estructural en cuestión es su estructura molecular; en el caso de las porciones de oro, su estructura atómica. Según Kripke es la posesión de este último tipo de propiedades las que determinan un género natural $\mathrm{y}$, por tanto, la referencia del término de género natural correspondiente. A este respecto se supone que hay una propiedad o un número reducido de propiedades estructurales constitutivas de (la pertenencia a) un género natural; el descubrimiento de estas propiedades es objeto de la investigación científica y hay buenas razones para creer que con respecto a algunos géneros naturales la ciencia ha descubierto tales propiedades.

A la hora de establecer el contraste entre su teoría de la referencia de los términos de género natural y la teoría descriptiva, Kripke supone que, según la teoría descriptiva, la referencia de los términos de género natural no vendría determinada mediante propiedades estructurales, sino mediante propiedades superficiales, a las que Kripke concibe como propiedades cualitativas o puramente generales.

La concepción de Mill acerca de los géneros naturales es diferente de la de Kripke, pero cabe alegar que no es una concepción completamente acorde con una teoría descriptiva de la referencia de los términos de género natural, tal como Kripke caracteriza a ésta. Ya hemos indicado que Mill concibe los géneros naturales como clases de entidades, más aún como clases que tienen "una existencia real en la naturaleza" (1843, p. 4), pero no todas las clases son géneros naturales. Los miembros de algunas clases son tales que comparten pocas propiedades, excepto las connotadas por el nombre que denota la clase; así, por ejemplo, los objetos pertenecientes a la clase de los objetos a los que se aplica el término "blanco" sólo son distinguidos por la blancura o "por propiedades conectadas de alguna manera con la blancura" (1843, p. 122), las cuales - ha de suponerse - serán igualmente connotadas por el término "blanco". Pero esto no ocurre en el caso de los miembros de los géneros naturales, por lo que el término "blanco" no es un término de género natural. Cabe considerar como la tesis principal de Mill acerca de los géneros naturales la de que éstos son clases que se diferencian entre sí por "una multitud indeterminada de propiedades no-derivables unas de otras" (Mill 1843, p. 126), ${ }^{10} \mathrm{de}$ tal manera que, aunque algunas de tales propiedades sean conocidas, muchas otras son desconocidas y muy probablemente continuarán siéndolo (Mill 1843, pp. 123 y 141). Hay especialmente dos rasgos recogidos en la afirmación recién citada que merecen ser tomados en consideración: la multiplicidad de las propiedades y su noderivabilidad.

10 Mill afirma acerca de las propiedades poseídas por los miembros de los géneros naturales que son un número "indefinido" (Mill 1843, pp. 126 y 703), un número infinito (Mill 1843, p. 579) e inagotables (Mill 1843, pp. 122 y 125). 
La concepción de los géneros naturales o, más precisamente, de sus miembros como constando de "una multitud indeterminada de propiedades" es difícil de encajar en la teoría kripkeana de los géneros naturales o, al menos, esa concepción no concuerda con los ejemplos puestos por Kripke en los que un género natural discernible sensorialmente es identificado con un género natural que, en la concepción de Kripke, constituye la esencia del primero (véase Kripke 1980, p. 138); piénsese en algunos de los famosos pares de ejemplos mencionados por Kripke, como agua $/ \mathrm{H}_{2} \mathrm{O}$ y oro/elemento con número atómico 79. En la teoría de Mill los géneros naturales son mucho más complejos de lo que parece asumirse en la teoría de Kripke. Ahora bien, puesto que en la teoría de Mill la pertenencia a un género natural viene determinada por una multitud de propiedades y muchas de ellas nos son desconocidas, éstas últimas no podrán figurar entre las propiedades connotadas por el término de género natural correspondiente. De aquí se sigue que los términos de género natural no poseen un rasgo central de los términos connotativos, pues, como ya indicamos, la relación existente entre la connotación y la denotación de los términos connotativos radica en que su connotación determina su denotación, pero esto no ocurrirá así en el caso de los términos de género natural, ya que las propiedades que connotamos mediante un término de género natural no serían todas aquellas que delimitarían el género natural y que, por tanto, permitirían diferenciarlo de otros, por lo que las propiedades connotadas por un término de género natural no constituirán condiciones necesarias y suficientes para la denotación o referencia del término. Como ya hemos indicado, según Mill, las clases cuyos miembros sólo comparten las propiedades connotadas por el término que denota la clase no son géneros naturales. Por tanto, con respecto a ese tipo de términos generales que denotan géneros naturales, es decir, los términos de género natural, su connotación no determina su denotación.

Conviene atender ahora a la no-derivabilidad a la que se aludía al caracterizar los géneros naturales como clases que se diferencian entre sí por "una multitud indeterminada de propiedades no-derivables unas de otras". Como hemos observado, Mill distingue entre las propiedades conocidas y las propiedades desconocidas de los (miembros de los) géneros naturales. Si aplicamos esta distinción al marco de Kripke, cabría alegar que las propiedades superficiales, o al menos la mayoría de ellas, estarán entre las propiedades conocidas, mientras que las propiedades desconocidas incluirán al menos algunas de las propiedades estructurales. Más aún, nuestro (supuesto) conocimiento de las propiedades estructurales es epistemológicamente más arriesgado que el de las propiedades superficiales y está abierto a revisión en mayor grado que el de estas últimas. Esto es así aunque Kripke hace hincapié en la revisabilidad de las propiedades superficiales con ciertos experimentos mentales en los que él nos pide, por ejemplo, que tomemos en consideración una situación contrafáctica de carácter epistemológico en la que imaginamos que en los lugares en los que hay minas de oro la atmósfera es tal que provoca en nosotros la ilusión óptica 
de que el oro tiene la apariencia de ser amarillo, pero una vez que se eliminan dichas condiciones atmosféricas descubrimos que el oro es azul (Kripke 1980, p. 118). Kripke propone experimentos mentales de este tipo con objeto de apuntalar su tesis de que no son las propiedades superficiales, sino las propiedades estructurales las que determinan (la pertenencia a) un género natural $\mathrm{y}$, por tanto, la referencia del término respectivo, pero Mill dista de entrar en estas cuestiones apelando simplemente a la distinción entre propiedades conocidas y propiedades desconocidas de (los miembros de) un género natural, de modo que tanto unas como otras intervienen en la delimitación de un género natural y, por ende, en la determinación de la referencia del término correspondiente.

En cualquier caso, un ámbito en el que cabe plantear la cuestión de la derivabilidad mutua de propiedades o su no-derivabilidad es el de la relación entre propiedades superficiales y propiedades estructurales. A este respecto conviene señalar que Kripke no afirma explícitamente la derivabilidad entre las propiedades superficiales de los miembros de un género natural y las propiedades concernientes a su estructura interna, si bien reconoce que aquéllas dependen de estás. Aunque Kripke no se expresa de manera demasiado clara acerca del vínculo de dependencia en cuestión, cabe suponer que él podría estar de acuerdo en que las leyes que involucran las propiedades estructurales de los miembros de los géneros naturales junto con ciertas condiciones iniciales referentes al entorno podrían explicar las propiedades superficiales correspondientes y en este sentido las propiedades superficiales serían derivables de las propiedades concernientes a la estructura interna. Más aún, en la medida en la que en la teoría de Kripke se acepta que estas últimas propiedades son propiedades esenciales de los miembros de un género natural, 11 e incluso constituyen la esencia del género natural, las propiedades estructurales en cuestión serán condiciones necesarias y suficientes de (la pertenencia a) los géneros naturales y, por tanto, de la determinación de la referencia de los términos de género natural. Esto ocurre en los casos correspondientes a lo que Kripke denomina "identificaciones téoricas" o "identidades teóricas", 12 es decir, enunciados de identidad (verdaderos) que expresan lo que hemos indicado anteriormente, a saber, la identidad entre un género natural discernible sensorialmente y un género natural que constituye la esencia del primero. Uno de los ejemplos puestos por Kripke es el del enunciado de identidad "Agua es $\mathrm{H}_{2} \mathrm{O}$ "; puesto que la propiedad de ser $\mathrm{H}_{2} \mathrm{O}$ constituye la esencia del agua, la propiedad de ser agua es derivable de la propiedad de ser $\mathrm{H}_{2} \mathrm{O}$, y la referencia del término "agua" es la misma que la del término " $\mathrm{H}_{2} \mathrm{O}$ ".

11 Las propiedades esenciales de una entidad son las propiedades que la entidad ha de tener en todos los mundos posibles en los que exista, dejando de lado algunas propiedades triviales, como la identidad consigo misma, y también la propiedad de la existencia (véase Kripke 1971, p. 151 y nota 11 de esa página).

12 Kripke usa indistintamente ambas expresiones, si bien emplea con mayor frecuencia la primera de ellas. 
No obstante, aceptando la existencia de una relación de derivabilidad de las propiedades superficiales con respecto a las propiedades estructurales, conviene señalar que esa relación no se dará previsiblemente entre las propiedades superficiales, o entre muchas de ellas. Sin embargo, llegados a este punto conviene señalar que Mill puntualiza, a pesar de su afirmación anteriormente citada, que la no-derivabilidad no concierne a todas las propiedades poseídas por los miembros de un género natural (1843, p. 123), si bien a la mayoría de ellas. Para establecer excepciones a la tesis de la no-derivabilidad habría que ejemplificarla con propiedades conocidas de un género natural, entre las cuales se encontrarán las que constituyen la connotación del término de género natural respectivo, o propiedades íntimamente relacionadas con ellas, pues Mill no puede dejar de considerar a los términos de género natural como términos generales y, en su teoría, éstos son términos connotativos.

Mill afirma:

De las propiedades innumerables, conocidas y desconocidas, que son comunes a la clase hombre, sólo una porción y naturalmente una porción muy pequeña, son connotadas por el nombre (Mill 1843, p. 127).

Un ejemplo que constituye la excepción a la no-derivabilidad es la propiedad de ser capaz de entender un lenguaje, que Mill considera como una propiedad exclusiva de los seres humanos, y aunque según Mill esta propiedad no pertenece a la connotación del término "hombre", se deriva de una de las propiedades pertenecientes a la connotación de dicho término (Mill 1843, p. 131), a saber, de la propiedad o del atributo de la racionalidad - véase el texto de Mill citado en la página 158 supra -. Un caso más general de la relación de derivabilidad se dará de manera trivial en el caso de un término definible mediante otros términos, pues la propiedad connotada por el primero se derivará de las propiedades connotadas por los términos que figuran en su definición, que pasarán así a constituir la propiedad connotada por el término, y esto ocurrirá con respecto a muchos términos connotativos, a saber, que la propiedad que constituye la connotación del término se derivará de las propiedades que constituyen tal propiedad. Así, empleando el ejemplo mencionado por Mill al comienzo de la sección 2., la propiedad connotada por el término "hombre", es decir, la propiedad de ser un hombre se derivaría de las propiedades de corporeidad, vida animal, racionalidad y de la propiedad de poseer una cierta forma externa. Según la teoría de Mill acerca de los términos generales, la conjunción de esas propiedades delimitaría la clase denotada por el término "hombre". Ahora bien, si el término "hombre" es un término de género natural, la clase en cuestión no debería venir especificada sólo por esas propiedades, sino que se requeriría "una multitud indeterminada de propiedades", muchas de ellas desconocidas y, por tanto, imposibles de figurar como propiedades a consignar en la connotación del término "hombre". 
De esta manera se constata una tensión entre la caracterización de los términos generales que Mill había formulado en el capítulo II del libro I (1843) y algunos de los componentes de la teoría de Mill acerca de ese peculiar tipo de términos generales que son los términos de género natural - Mill comienza a ocuparse de los géneros naturales a partir del capítulo VII del libro I -. Esto le lleva en ocasiones a hacer afirmaciones acerca de los géneros naturales, y de los términos de género natural, que entran en conflicto entre sí al no tomar en debida consideración la concepción que él mismo ha formulado acerca de los géneros naturales. Así en una ocasión Mill llega a afirmar que "el nombre de cada clase [de cada clase que constituye un género natural] debe connotar necesariamente suficientes de las propiedades para fijar los límites de la clase" (Mill 1843, p. 141). Ahora bien, a este respecto conviene señalar, por una parte, y como ya indicamos, que Mill alega que las clases cuyos miembros sólo comparten las propiedades connotadas por el nombre o término que denota la clase no son géneros naturales y, por otra, que la tesis principal de Mill acerca de los géneros naturales - pasando por alto la cuestión de la noderivabilidad - es que por un género natural se entiende "una de las clases que se distinguen de otras no por una o por unas pocas propiedades definidas, sino por una multitud desconocida de propiedades" (Mill 1843, p. 703; en el mismo sentido véase, por ejemplo, pp. 123, 126 y 579). Si los géneros naturales se diferencian entre sí por una multitud de propiedades, algunas conocidas y otras muchas desconocidas, es este conjunto global de propiedades el que delimitaría el género natural $\mathrm{y}$, por tanto, determinaría la referencia del término de género natural correspondiente, y no sólo el pequeño número de propiedades conocidas que puedan constituir la connotación del término. Las propiedades connotadas por un término de género natural serán las más obvias o aquellas de entre las que se han descubierto - o conocido - que se consideren más importantes. Pero los miembros de un género natural tienen otras propiedades que no se derivan de las propiedades que constituyen la connotación del término de género natural correspondiente y que contribuirán igualmente a la delimitación del género natural y, por ende, a la determinación de la referencia del término de género natural respectivo.

Ahora bien, si esto es así, la teoría semántica de los términos de género natural que cabe atribuir a Mill no es una teoría descriptiva tal como Kripke caracteriza este tipo de teoría, y, a pesar de que, como hemos indicado, Mill no es completamente consecuente a este respecto, cabría cuestionar desde una concepción de los géneros naturales como la de Mill, que "un predicado tal como 'ser humano' se define como la conjunción de ciertas propiedades que proporcionan condiciones necesarias y suficientes para la humanidad" (Kripke 1980, p. 127), donde por "propiedades" habrá de entenderse "propiedades conocidas", que supuestamente figurarían en la definición del término/predicado. Por la misma razón cabría cuestionar desde dicha concepción que los términos "vaca" y "tigre", en la medida en que denoten géneros naturales, sean "abreviaturas de la conjunción de propiedades mediante las cuales 
los podría definir un diccionario" (Kripke 1980, p. 128). A este respecto Kripke no ha tomado suficientemente en consideración la concepción de los géneros naturales sostenida por Mill - al igual que le ocurre al propio Mill - y le atribuye una teoría de los términos de género natural que discrepa de la que este autor ha formulado. Posiblemente la errónea interpretación de Kripke se debe a una cierta inconsecuencia por parte de Mill, que hemos señalado. Este autor concibe los términos de género natural como términos generales $\mathrm{y}$, por tanto, como términos connotativos, por lo que deberían cumplir el principio de que la connotación determina la denotación; pero la teoría genérica de Mill acerca de los términos generales no coincide, como hemos alegado, con la sostenida por el propio Mill al ocuparse de ese tipo de términos generales que son los términos de género natural, a los que no debería aplicarse el anterior principio de que la connotación determina la denotación.

\section{Referencias bibliográficas}

Fernández Moreno, L. (2006): La referencia de los nombres propios, Madrid, Trotta.

FERNÁNDEZ MORENO, L. (2009): "Sentido, referencia y representación lingüística en Frege", en P.F. Martínez-Freire (ed.) Representaciones, Suplemento 14 de Contrastes. Revista Internacional de Filosofía, pp. 31-48.

KrIPKE, S. (1971): "Identity and Necessity", en M. Munitz (ed.), Identity and Individuation, Nueva York, New York University Press, pp. 135-164.

KRIPKE, S. (1980): Naming and Necessity, Oxford, Blackwell. Reimp. revisada y con prefacio añadido de "Naming and necessity", en D. Davidson y G. Harman (eds.), Semantics of Natural Language, Reidel, Dordrecht, 1972.

MiLl, J.S. (1843): A System of Logic Ratiocinative and Inductive, edición a cargo de J.M. Robson, Toronto, University of Toronto Press, 1973.

Russell, B. (1912): The Problems of Philosophy, Londres, Williams Norgate. Citada por la siguiente edición: The Problems of Philosophy, Oxford, Oxford University Press, 1959.

Salmon, N. (2005): Reference and Essence. Amherst: Prometheus Books; $2^{\mathrm{a}}$ ed. ampliada; $1^{\text {a }}$ ed., Princeton, Princeton University Press, 1981.

Searle, J. (1969): Speech Acts. An Essay in the Philosophy of Language, Cambridge, Cambridge University Press.

Luis Fernández Moreno

Dpto. de Lógica y Filosofía de la Ciencia

Universidad Complutense de Madrid

luis.fernandez@filos.ucm.es 